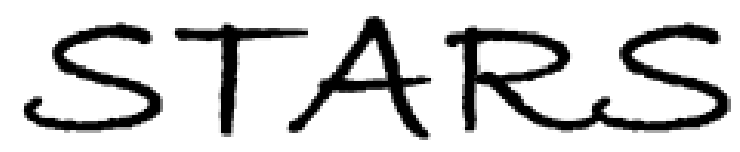

University of Central Florida

STARS

Faculty Bibliography 1990s

Faculty Bibliography

$1-1-1991$

\title{
Geometry Of Quantum Evolution And The Coherent State
}

Subir K. Bose

University of Central Florida

Binayak Dutta-Roy

University of Central Florida

Find similar works at: https://stars.library.ucf.edu/facultybib1990

University of Central Florida Libraries http://library.ucf.edu

This Article is brought to you for free and open access by the Faculty Bibliography at STARS. It has been accepted for inclusion in Faculty Bibliography 1990s by an authorized administrator of STARS. For more information, please contactSTARS@ucf.edu.

\section{Recommended Citation}

Bose, Subir K. and Dutta-Roy, Binayak, "Geometry Of Quantum Evolution And The Coherent State" (1991). Faculty Bibliography 1990s. 203.

https://stars.library.ucf.edu/facultybib1990/203

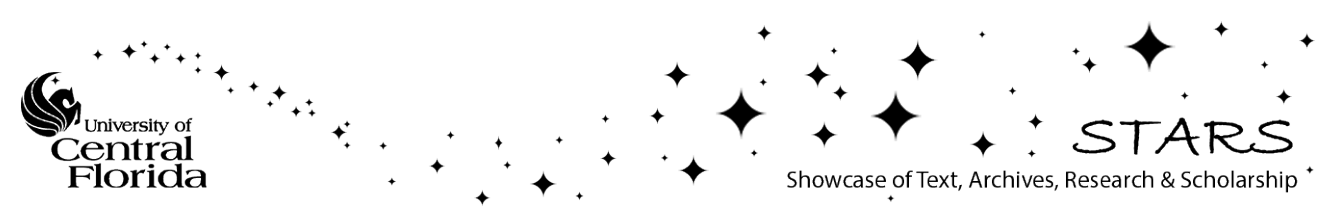




\title{
PHYSICAL REVIEW A
}

\author{
ATOMIC, MOLECULAR, AND OPTICAL PHYSICS
}

\section{Geometry of quantum evolution and the coherent state}

\author{
Subir K. Bose and Binayak Dutta-Roy* \\ Department of Physics, University of Central Florida, Orlando, Florida 32816
}

(Received 5 November 1990)

\begin{abstract}
The geometric approach to quantum mechanics initiated by Berry's remarkable discovery [Proc. R. Soc. London Ser. A 392, 45 (1984)] of the anholonomy of the phase of the wave function and subsequent developments leading to a recent reformulation of the geometry of quantum evolution by Anandan and Aharonov [Phys. Rev. Lett. 65, 1697 (1990)] is shown to find an explicit and suggestive realization through the coherent-state representation.
\end{abstract}

\section{INTRODUCTION}

Upon the adiabatic variation of parameters, $\left\{X_{i}\right\}$, in the Hamiltonian of a system through a closed path $C$, the wave function was found by Berry ${ }^{1}$ to acquire, apart from the familiar dynamical phase $\left(\exp \left[(-i / \hbar) \int E(t) d t\right]\right)$ associated with the time evolution of the state being so transported with instantaneous eigen energy $E(t)$, an additional phase $(\exp [i \gamma(C)])$ depending only on the geometry of the parameter space and the path traversed therein. Simon ${ }^{2}$ gave a topological meaning to the Berry's phase as the holonomy of a complex line bundle. Aharonov and Anandan ${ }^{3}$ showed how any closed path in the projective Hilbert space (of state vectors modulo phases) has a geometric phase associated with it and from this point of view the underlying parameter space has but a secondary role, and adiabaticity is not an essential ingredient. Samuel and Bhandari ${ }^{4}$ properly emphasizing the metric on the projective Hilbert space $(\mathcal{P})$ put the concept of the geometric phase in an even more general setting which would apply to essentially any type of quantum evolution conceivable, liberated from the constraints of having to be adiabatic, cyclic, or even unitary.

In a recent paper Anandan and Aharonov ${ }^{5}$ have related the metric in projective Hilbert space, $\mathcal{P}$, with the time-energy uncertainty product. They consider the evolution of a nonstationary state $|\psi(t)\rangle$ governed by the Schrödinger equation

$$
i \hbar \frac{d}{d t}|\psi(t)\rangle=H(t)|\psi(t)\rangle,
$$

enabling a Taylor-series expansion,

$$
\begin{aligned}
|\psi(t+d t)\rangle= & |\psi(t)\rangle-\frac{i}{\hbar} d t H|\psi(t)\rangle \\
& -\frac{(d t)^{2}}{2 \hbar}\left[i \frac{d H}{d t}|\psi(t)\rangle+\frac{1}{\hbar} H^{2}|\psi(t)\rangle\right] \\
& +O\left((d t)^{3}\right),
\end{aligned}
$$

from which follows

$$
|\langle\psi(t) \mid \psi(t+d t)\rangle|^{2}=1-\frac{(d t)^{2}(\Delta E)^{2}}{\hbar^{2}}+O\left((d t)^{3}\right),
$$

where

$$
(\Delta E)^{2}=\left\langle\psi\left|H^{2}\right| \psi\right\rangle-\langle\psi|H| \psi\rangle^{2} .
$$

On the other hand, a natural measure ${ }^{6}$ of the squared "distance" between two nearby quantum states is the deviation from unity of the square modulus of their scalar product (their overlap), namely, $\Delta s_{12}^{2}=1-|\langle 1 \mid 2\rangle|^{2}$, and several authors ${ }^{7-10}$ have considered similar definitions. Anandan and Aharonov, ${ }^{5}$ comparing Eq. (3a) with the definition of the Fubini-Study metric, ${ }^{7}$ arrive at the interesting correlation

$$
d s=\Delta E d t / \hbar,
$$

which they paraphrase through the evocative statement that $\Delta E / \hbar$ is the "magnitude of the velocity of the system in the projective Hilbert space" providing thereby a new geometric meaning to the uncertainty in energy for a quantum system.

It is important to emphasize that for the realization of the interpretation advocated by Anandan and Aharonov

(C) 1991 The American Physical Society 
it is essential to consider nonstationary states of a quantum system, rather than eigenstates of the Hamiltonian as considered by Berry, as in the latter case $\Delta E \rightarrow 0$ whereas $d t \rightarrow \infty$ so that $d s$ is finite and nonzero, and this singular limit is not suitable for the study of the physical manifestations of the Fubini-Study metric. The objective of the present investigation is to explore the contention that the coherent states provide a particularly convenient framework for the illustration of the geometry of quantum evolution in this context. With this end in view the two archetypal examples of a spinning particle in a time-varying magnetic field and that of a parametrically varying oscillator are considered. In each case the method of presentation followed is to start with the underlying bosonic representation of the Heisenberg-Weyl algebra and then to set up the relevant Heisenberg equations of motion. This has the added advantage in that these equations are formally identical to Hamilton's equations of classical mechanics, and the relationship with the corresponding classical anholonomy associated with the Hannay angles $^{11-13}$ is quite transparent. Furthermore, as we shall see in the case of the particle in a magnetic field the generalization from the case of spin- $\frac{1}{2}$ to that of general angular momentum is easily accomplished. In this framework we determine the Berry phases and then go on to construct the coherent states and set up the Fubini-Study metric for each system in order to illustrate the geometric interpretation due to Anandan and Aharonov. We use the notion of adiabaticity even though it is not essential to this approach in order to maintain contact with earlier studies, as also for ease of explicit solution and interpretation.

\section{SPIN IN A MAGNETIC FIELD}

Consider a particle of spin $\frac{1}{2}$ and magnetic moment $\mu$ precessing in a homogeneous though time-dependent magnetic field $\mathbf{B}(t)$ described by the Hamiltonian

$$
H=-\boldsymbol{\mu} \cdot \mathbf{B}(t)=-\mu \boldsymbol{\sigma} \cdot \mathbf{B}(t),
$$

where $\mathbf{B}(t)$ is taken to be slowly varying (in comparison to the Larmor frequency $\left.\omega_{L}=\mu B / \hbar\right)$ keeping its magnitude fixed but gyrating with angular frequency $\epsilon$ about the $z$ axis, sweeping out a cone of semivertical angle $\theta$, whence

$$
\mathbf{B}(t)=B(\sin \theta \cos (\epsilon t), \sin \theta \sin (\epsilon t), \cos \theta) .
$$

In accordance with the program outlined in the Introduction we search for a bosonic realization:

$\left[a_{i}, a_{j}\right]=0=\left[a_{i}^{\dagger}, a_{j}^{\dagger}\right],\left[a_{i}, a_{j}^{\dagger}\right]=\delta_{i j} \quad$ with $i, j= \pm$,

such that

$$
\sigma_{z}=\left(a_{+}^{\dagger} a_{+}-a_{-}^{\dagger} a_{-}\right)
$$

and

$$
\sigma_{ \pm}=\frac{1}{2}\left(\sigma_{x} \pm i \sigma_{y}\right)=a_{ \pm}^{\dagger} a_{\mp} .
$$

The Hamiltonian expressed in terms of $a_{ \pm}$and $a_{ \pm}^{\dagger}$ can be put into a convenient form

$$
H=-\mu B\left(b_{+}^{\dagger} b_{+}-b_{-}^{\dagger} b_{-}\right),
$$

through a canonical, albeit time-dependent transformation

$$
\begin{aligned}
& b_{+}=+\cos \left(\frac{1}{2} \theta\right) e^{i \epsilon t / 2} a_{+}+\sin \left(\frac{1}{2} \theta\right) e^{-i \epsilon t / 2} a_{-}, \\
& b_{-}=-\sin \left(\frac{1}{2} \theta\right) e^{i \epsilon t / 2} a_{+}+\cos \left(\frac{1}{2} \theta\right) e^{-i \epsilon t / 2} a_{-},
\end{aligned}
$$

which corresponds to a rotation bringing the $z$ axis along the instantaneous direction of the magnetic field. The Heisenberg equations of motion are accordingly given by

$$
\begin{aligned}
\frac{d}{d t} b_{+} & =-\frac{i}{\hbar}\left[b_{+}, H\right]+\frac{\partial b_{+}}{\partial t} \\
& =+i \omega_{L} b_{+}+i \frac{1}{2} \epsilon(\cos \theta) b_{+}-i \frac{1}{2} \epsilon(\sin \theta) b_{-}, \\
\frac{d}{d t} b_{+} & =-\frac{i}{\hbar}\left[b_{-}, H\right]+\frac{\partial b_{-}}{\partial t} \\
& =-i \omega_{L} b_{-}-i \frac{1}{2} \epsilon(\cos \theta) b_{-}-i \frac{1}{2} \epsilon(\sin \theta) b_{+} .
\end{aligned}
$$

These can readily be solved exactly, but in order to find the Berry phase it suffices to work to the first order of adiabaticity (here $\epsilon / \omega_{L}$ ), and accordingly one obtains

$b_{ \pm}(T) \sim \exp \left[ \pm i \omega_{L} T \mp i \epsilon T(1-\cos \theta) / 2\right] b_{ \pm}(0)$,

corresponding to a round trip of the magnetic field ( $t=T=2 \pi / \epsilon$ ) giving the Berry phase in the Heisenberg picture, with all its geometric connotations involving the relevant solid angle $[\Omega(C)=2 \pi(1-\cos \theta)]$ in parameter space, over and above the dynamic phase ${ }^{14}$ given by $\omega_{L} T$. The result obtained here can easily be converted to the more familiar form in terms of the phase of the state vector by going from the Heisenberg to the Schrödinger picture. Furthermore, the generalization from the case of spin- $\frac{1}{2}$ to arbitrary angular momentum $j$ is easily accomplished by taking recourse to the well-known Schwinger construction $^{15}$

$$
|j m\rangle=\frac{\left(b_{+}^{\dagger}\right)^{j+m}\left(b_{-}^{\dagger}\right)^{j-m}}{\sqrt{(j+m) !(j-m) !}}|0\rangle,
$$

and the corresponding Berry phase, $-m \Omega(C)$, can be read off through an inspection of Eqs. (10) and (11).

In order to illustrate the concepts developed by Anandan and Aharonov ${ }^{5}$ it behooves us to consider the angular-momentum coherent state ${ }^{16}$

$$
\begin{aligned}
|\xi\rangle & =N e^{\xi J}|j,-j\rangle \\
& =\frac{1}{\left(1+|\xi|^{2}\right)^{j}} \sum_{n=0}^{2 j} \xi^{n}\left[\begin{array}{c}
2 j \\
n
\end{array}\right]^{1 / 2}|j,-j+n\rangle,
\end{aligned}
$$

where $N$ is the normalization constant, $\xi$ a complex number specifying the state, and $\left(\begin{array}{l}2 j \\ n\end{array}\right)$ is the binomial combinatoric $(2 j) ! / n !(2 j-n)$ ! For a particle in such a state with gyromagnetic ratio $g$ placed in a magnetic field $B$ the spread in energy is easily calculated to be

$$
\Delta E=g B|\xi| \hbar \sqrt{2 j} /\left(1+|\xi|^{2}\right) .
$$

The coherent state [Eq. (12)] may be reexpressed in terms of the Heisenberg-Weyl operators to yield 


$$
|\xi\rangle=\frac{1}{\left(1+|\xi|^{2}\right)^{j}} \frac{1}{\sqrt{(2 j) !}}\left(\xi b_{+}^{\dagger}+b_{-}^{\dagger}\right)^{2 j}|0\rangle,
$$

wherein implementing Eq. (10) the time development of the wave packet is given by

$$
\begin{aligned}
|\xi\rangle_{T}= & e^{2 i j \omega_{L} T-i \epsilon j(1-\cos \theta) T} \\
& \times\left|\xi \exp \left[-2 i \omega_{L} T+i \epsilon T(1-\cos \theta)\right]\right\rangle .
\end{aligned}
$$

Thus even after a cycle (a time $T=2 \pi / \epsilon$ later when the Hamiltonian will have returned to its starting value) the state shall gain not only a phase, but more importantly, unlike what obtains for a stationary state, shall have changed to a different state and shall have moved in projective Hilbert space. The geometry of this movement is best described by considering the Fubini-Study metric given by

$$
\begin{aligned}
(d s)^{2} & =1-|\langle\xi+d \xi \mid \xi\rangle|^{2} \\
& =\frac{2 j}{\left(1+\xi_{1}^{2}+\xi_{2}^{2}\right)^{2}}\left[\left(d \xi_{1}\right)^{2}+\left(d \xi_{2}\right)^{2}\right],
\end{aligned}
$$

where $\xi_{1}$ and $\xi_{2}$ are the real and imaginary parts of $\xi$, and the metric above is that for the surface of a sphere of radius $2 j$ expressed in isothermal co-ordinates ${ }^{17}$, and can be brought to the more familiar form through the substitution $\xi=\exp (i \Phi / 2) \tan (\Theta / 2)$, where $\Theta$ and $\Phi$ are the usual co-latitude and azimuth angles.

One could go on to discuss this same problem in the format of the transport of a more general wave packet where different $j$ 's are also superposed centered around a given value of the angular momentum. Such a state is indeed available to us, ${ }^{18}$ having been constructed through the strategem of a noncompact extension of the SU(2) group, ${ }^{15}$ and reads

$$
\begin{aligned}
|\rho, v\rangle= & \left\{\cosh \left[|v|(1+|\rho|)^{2}\right]\right\}^{-1 / 2} \\
& \times \sum_{j=0}^{\infty} \sum_{m=-j}^{+j} \frac{\rho^{j-m} v^{j}}{\sqrt{(j+m) !(j-m) !}}|j, m\rangle,
\end{aligned}
$$

which assumes an elegant appearance when expressed in terms of the Heisenberg-Weyl operators:

$$
|\eta, \zeta\rangle=\left[\cosh \left(|\eta|^{2}+|\zeta|^{2}\right)\right]^{-1 / 2} \cosh \left(\eta a_{+}^{\dagger}+\zeta a_{-}^{\dagger}\right)|0\rangle,
$$

where $v=\eta^{2}$ and $\rho=\zeta / \eta$. The evolution of this wave packet has already been considered earlier ${ }^{13}$ in order to establish the relationship with the Hannay angle.

\section{THE GENERALIZED PARAMETRIC HARMONIC OSCILLATOR}

The general time-dependent quadratic Hamiltonian with one freedom, appropriately symmetrized, is given by

$$
H(t)=Z(t) \frac{p^{2}}{2 m}+\frac{\omega_{0}}{2} \boldsymbol{Y}(t)(p q+q p)+X(t) \frac{1}{2} m \omega_{0}^{2} q^{2} .
$$

Introducing the annihilation (and creation) operator through $a=\left(p-i m \omega_{0} q\right) / \sqrt{2 m \omega_{0} \hbar} \quad$ and $a^{\dagger}=(p$ $\left.\left.+i m \omega_{0} q\right) / \sqrt{2 m \omega_{0} \hbar}\right]$, and recognizing that the system
Hamiltonian is obtainable from that for the simple oscillator by a scaling and rotation in phase space, a canonical transformation of the Bogoliubov-Valatin type, namely,

$$
\begin{aligned}
b= & \frac{1}{2}\left(\sqrt{Z / \kappa}+\sqrt{\kappa / Z}+i \frac{Y}{\sqrt{\kappa Z}}\right) a \\
& +\frac{1}{2}\left(\sqrt{Z / \kappa}-\sqrt{\kappa / Z}-i \frac{Y}{\sqrt{k Z}}\right) a^{\dagger},
\end{aligned}
$$

where $\kappa=\left(X Z-Y^{2}\right)^{1 / 2}$, suggests itself, and brings the Hamiltonian to the form

$$
H=\hbar \omega(t)\left(b^{\dagger} b+\frac{1}{2}\right) .
$$

Here $\omega(t) \equiv \kappa(t) \omega_{0}$ is the "instantaneous frequency" and the operator $b$ (and $b^{\dagger}$ ) is explicitly time dependent through the "externally" varied parameters. The Heisenberg equations of motion for the system are thus

$$
\begin{aligned}
\frac{d b}{d t}= & -\frac{i}{\hbar}[b, H]+\frac{\partial b}{\partial t} \\
= & -i \omega b+i\left[\frac{Z}{2 \kappa} \frac{d}{d t}\left[\frac{Y}{Z}\right]\right] b \\
& -i\left[\frac{Z}{2 \kappa} \frac{d}{d t}\left[\frac{Y-i \kappa}{Z}\right]\right] b^{\dagger}, \\
\frac{d b^{\dagger}}{d t}= & -\frac{i}{\hbar}\left[b^{\dagger}, H\right]+\frac{\partial b^{\dagger}}{\partial t} \\
= & +i \omega b^{\dagger}-i\left[\frac{Z}{2 \kappa} \frac{d}{d t}\left[\frac{Y}{Z}\right]\right] b^{\dagger} \\
& +i\left[\frac{z}{2 \kappa} \frac{d}{d t}\left[\frac{Y+i \kappa}{Z}\right]\right] b,
\end{aligned}
$$

and the terms representing the coupling between the creation and the annihilation operators contributing, as before, to second order in adiabaticity, can be disregarded for the purpose of calculating the Berry phase, and the approximate solution

$$
\begin{aligned}
b(t) \sim \exp & \left(-i \int \omega(t) d t\right] \\
& \times \exp \left[\frac{i \omega_{0}}{2} \int \frac{Z}{\omega} \frac{d}{d t}\left[\frac{Y}{Z}\right] d t\right] b(0)
\end{aligned}
$$

suffices. Contact with the usual expression ${ }^{12,19}$ for the phase can be established as before by going over to the Schrödinger picture and furthermore, by rewriting the second phase factor in Eq. (23) as a line integral in parameter space by simply noting that

$$
\frac{d}{d t}=\frac{d \mathbf{X}}{d t} \cdot \nabla
$$

where $\mathrm{X}$ stands for $(X, Y, Z)$ and $\nabla$ is the gradient operator in parameter space.

To illustrate the motion in the projective Hilbert space it is again appropriate to construct the corresponding coherent state: 


$$
|\beta\rangle=e^{-|\beta|^{2}} \sum_{n=0}^{\infty} \frac{\beta^{n}}{\sqrt{n !}}|n\rangle=e^{\beta b^{\dagger}-\beta^{*} b}|0\rangle,
$$

which is an eigenstate of the operator $b$ obtained from the primitive operator $a$ through a canonical transformation [Eq. (20)] of the generic form

$$
b=\lambda(t) a+\varsigma(t) a^{\dagger},
$$

with

$$
|\lambda(t)|^{2}-|\varsigma(t)|^{2}=1 .
$$

These states are the squeezed coherent states ${ }^{20}$ as they involve viz á vis the corresponding coherent states for the primitive $a$ operator, a scaling and rotation (a squeezing in one direction) in phase space. The time development of this state is easily obtained by implementing Eq. (23) in Eq. (25) to yield

$$
\begin{aligned}
|\beta\rangle_{t}=\mid \beta \exp [ & +i \int \omega(t) d t \\
& \left.\left.\quad-i \frac{\omega_{0}}{2} \int \frac{Z}{\omega} \nabla\left(\frac{Y}{Z}\right) \cdot d \mathbf{R}\right]\right\rangle,
\end{aligned}
$$

to first order in adiabaticity. Once again the change in the state is to be noted (in contrast to the appearance of a phase for the stationary state); and in order to depict the corresponding motion in the projective Hilbert space the underlying Fubini-Study metric is easily determined [using Eq. (25) and introducing $\beta_{1}$ and $\beta_{2}$ for the real and imaginary parts of $\beta$ ]:

$$
(d s)^{2} \equiv 1-|\langle\beta+d \beta \mid \beta\rangle|^{2}=\left(d \beta_{1}\right)^{2}+\left(d \beta_{2}\right)^{2},
$$

which corresponds to a flat Euclidean space. If, however, the "distance" had been expressed in terms of the "primitive coordinates" obtained from the eigenvalue $\alpha$ of the operator $a$ then the associated squeezing and rotation would be manifest in the metric. It is perhaps appropriate to point out that our definition of the distance, here and earlier, differs from that of Anandan and Aharonov ${ }^{5}$ by an overall factor of 2 . This has been done to maintain consistency with the earlier definition given by Berry ${ }^{6}$ as the real symmetric part of the quantum geometric tensor, and more importantly, in order to correspond to the intuitive notion of velocity in phase space as shall be illustrated by a simple example. Consider a simple harmonic oscillator described by a Hamiltonian

$$
H=\frac{p^{2}}{2 m}+\frac{1}{2} m \omega_{0}^{2} x^{2}=\hbar \omega_{0}\left(a^{\dagger} a+\frac{1}{2}\right),
$$

and the associated coherent state specified by the complex number $\alpha$ with real and imaginary parts $\alpha_{1}$ and $\alpha_{2}$, then putting $\alpha(0)=|\alpha(0)| \exp (i \vartheta)$ the time development of the state would be characterized by $\alpha(t)$ $=\alpha(0) \exp (i \omega t)$ and hence with $(d s)^{2}=\left(d \alpha_{1}\right)^{2}+\left(d \alpha_{2}\right)^{2}$ and $\alpha_{1}=|\alpha(0)| \cos (\omega t+\vartheta)$ and $\alpha_{2}=|\alpha(0)| \sin (\omega t+\vartheta)$, the velocity in the projective Hilbert space is $d s / d t=\omega|\alpha|$ which also agrees with the velocity in phase space of a point moving with angular velocity $\omega$ along a circle of radius $|\alpha|$. And of course the velocity is in agreement, as it must, with that given by the spread of energy corresponding to this state, namely, $\Delta E=\hbar \omega|\alpha|$.

\section{CONCLUSION}

Thus we conclude that the main contention of Anandan and Aharonov's recent paper ${ }^{5}$ regarding the geometry of quantum evolution, its relation with the uncertainty principle and with the Fubini-Study metric in projective Hilbert space can be explicitly and simply realized in the context of coherent states. This is illustrated by considering the problems of the spin in a magnetic field and the generalized parametric oscillator.
${ }^{*}$ On leave from the Saha Institute of Nuclear Physics, Calcutta, India.

${ }^{1}$ M. V. Berry, Proc. R. Soc. London, Ser. A 392, 45 (1984).

${ }^{2}$ B. Simon, Phys. Rev. Lett. 51, 2167 (1983).

${ }^{3}$ Y. Aharonov and J. Anandan, Phys. Rev. Lett. 58, 1593 (1987).

${ }^{4}$ J. Samuel and R. Bhandari, Phys. Rev. Lett. 60, 2339 (1988).

5 J. Anandan and Y. Aharonov, Phys. Rev. Lett. 65, 1697 (1990).

${ }^{6}$ See, for example, M. V. Berry, in Geometric Phases in Physics, edited by A. Shapere and F. Wilczek (World Scientific, Singapore, 1989), p. 14.

${ }^{7}$ S. Kobayashi and K. Nomizu, Foundations of Differential Geometry (Interscience, New York, 1969).

${ }^{8}$ D. A. Page, Phys. Rev. A 36, 3479 (1987).

9J. P. Provost and G. Vallee, Commun. Math. Phys. 76, 289 (1980).

${ }^{10}$ C. Bouchiat and G. W. Gibbons, J. Phys. (Paris) 49, 187 (1988).

11J. Hannay, J. Phys. A 18, 221 (1985).
${ }^{12}$ M. V. Berry, J. Phys. A 18, 15 (1985).

${ }^{13}$ G. Ghosh and B. Dutta-Roy, Phys. Rev. D 37, 1709 (1988).

${ }^{14}$ See, for example, A. Messiah, Quantum Mechanics (NorthHolland, Amsterdam, 1961), Vol. 1, p. 319; note the insertion of an additional phase $\mp i \epsilon T / 2$ to conform to the eigenstate of the instantaneous Hamiltonian as reference.

15J. Schwinger, in Quantum Theory of Angular Momentum, edited by L. C. Beidenharn and H. Van Dam (Academic, New York, 1965).

16J. M. Radcliffe, J. Phys. A 4,-313 (1971).

${ }^{17}$ See M. P. do Carmo, Differential Geometry of Curves and Surfaces (Prentice-Hall, 1976), p. 227.

${ }^{18}$ D. Bhaumik, T. S. Nag, and B. Dutta-Roy, J. Phys. A 8, 1868 (1975).

19J. M. Cervero and J. D. Lejarreta, J. Phys. A 22, L663 (1989).

${ }^{20}$ See, for example, J. Perina, Coherence of Light (Van Nostrand, New York, 1971). 